\title{
Numerical Study of Motion of Sun-Grazing Comet C/2011 W3 (Lovejoy) Affected by Outgassing, General Relativity, and Oblateness Correction Forces
}

\author{
R. W. Wibowo, B. Dermawan, T. Hidayat, D. \\ Mandey, I. N. Huda, I. Tampubolon \\ Faculty of Mathematics \& Natural Sciences \\ Bandung Institute of Technology \\ Bandung, Indonesia \\ e-mail: ridlo.w.wibowo@gmail.com
}

\author{
J. A. Utama \\ Faculty of Mathematics \& Natural Sciences Education \\ Indonesia University of Education \\ Bandung, Indonesia
}

\begin{abstract}
Among comets there are several groups of Sungrazers. Sun-grazing comets are comets whose motions encounter very closely to the Sun and may eventually plunge into the Sun. Sun-grazing comet C/2011 W3 (Lovejoy) was not expected to survive during close encounter with the Sun on December 16, 2011 due to the extreme conditions in the corona. We study numerically orbit of the comet during this event. We developed numerical codes of additional forces (i.e. cometary outgassing, general relativity, and the oblateness/quadrupole moment of the Sun) and incorporated them into the new integrator package REBOUND. These additional forces yield small changes in motion of the comet near the event. As expected, at about the event we find that contributions of the general relativity and the outgassing forces to the comet's motion are larger than the oblateness correction of the Sun. This study also shows that the role of the outgassing force may shift event of the next encounter for at least about a hundred days.
\end{abstract}

Keywords-comet C/2011 W3 (Lovejoy), cometary dynamics, N-body.

\section{INTRODUCTION}

Comet C/2011 W3 (Lovejoy) is a Kreutz Sun-grazer, long period comet which was discovered in November 2011 by Terry Lovejoy [1]. The comet Lovejoy was expected not to survive on its perihelion passage on 16 December 2011 at 00:17 UTC due to tidal force and coronal activity. However, this comet re-appeared from behind the Sun then the nucleus disintegrated $\sim 2$ days after perihelion passage as observed by STEREO spacecraft [1].

The majority of the Sun-grazer comets are small fragments (meter-sized objects) that can only be observed during close encounter with the Sun (by Solar observatory spacecraft). Kreutz Sun-grazer family consist of comets on similar highly inclined and high-eccentric orbits. Some of them are prominent comet in history such as Ikeya-Seki (C/1665 S1) and Great Comet of 1882 (C/1882 R1). Dynamical behavior of the fragmentation event which producing this family have been studied by [2].

We chose this comet as study case of a very eccentric orbit with REBOUND integrator [3]. Orbit of comet Lovejoy has very small perihelion distance, so that the general relativity play important role to the equations of motion near the encounter event. We also implemented oblateness correction force and symmetric outgassing parameter to this integrator. We improved this integrator with those three additional forces to the equations of motion.

\section{INITIAL DATA AND INTEGRATION SCHEME}

Orbital element of comet C/2011 W3 (Lovejoy) at JD 2455904.5 (Dec 9th, 2011), a week before perihelion passage was used as initial data. All planets from Mercury to Neptune, Moon, and three massive asteroid were included as perturbing bodies of the comet. These data provided in the JPL Small-Body Database, NASA ${ }^{1}$.

A new Integrator with Adaptive Step-size control, 15th order (IAS15) [4], first introduced in REBOUND was used. IAS15 (based on RADAU15 algorithm) is off a very high order, nonsymplectic that can handle arbitrary (velocity dependent) forces and is in most cases accurate down to machine precision. This integrator is convenient to be used for very eccentric orbit, and also because we will add several velocity dependent forces in the equations of motion. As initial we used time step of 0.001 days and accuracy parameter of $10^{-3}$. A short-time integration of 2000 years is chosen to study the effect of the additional forces. A set of equations of motion for the comet (e.g. index $j$ ) in Cartesian coordinate are expressed by Newtonian equation of motion

$$
\ddot{\boldsymbol{r}}_{j}=-G \sum_{i=1}^{N} \frac{m_{i}}{\left|\boldsymbol{r}_{i}-\boldsymbol{r}_{j}\right|^{3}}\left(\boldsymbol{r}_{i}-\boldsymbol{r}_{j}\right)+\boldsymbol{P}_{e x t}
$$

Where $\boldsymbol{r}$ denotes the radius vector, dots are the usual derivation with respect to time, $G$ is the gravitational constant, $N$ is the number of perturbing bodies (index $i$ ), and $\boldsymbol{P}_{\text {ext }}$ is acceleration term from the additional forces.

\section{IMPLEMENTATION OF ADDITIONAL FORCES}

\section{A. REBOUND Integrator Package}

We used REBOUND as integrator, an open-source multipurpose $N$-body code for collisional dynamics (http://github.com/hannorein/rebound). We used this code due to high modularity and can be customized easily. It can be used to work on a wide variety of different problems in

\footnotetext{
${ }^{1}$ http://ssd.jpl.nasa.gov/horizons.cgi
} 
astrophysics and beyond [3]. It is also support parallelization with MPI and OpenMP. Many examples of the typical problems provided in the examples/ directory. Each example comes with a problem file (problem.c) and makefile (Makefile) as template used in this code. We can easily include the additional force in the integration process by initialize it in the problem file.

$$
\text { problem_additional_forces }=\text { additional_force; }
$$

When we compile our problem (make), REBOUND will read the additional_force function and implement it after calculating all gravitational forces. Total acceleration from gravity and additional forces will be used in the equations of motion.

\section{B. Relativistic correction}

Our purpose is to review how general relativity affects the orbital dynamics of Sun-grazer comets. For the simplified case where only one object (e.g. the Sun) contributes and in the reference frame of this central, massive object with mass $M$, the relativistic correction would become

$$
\Delta \ddot{\boldsymbol{r}}_{j}=\frac{G M}{r^{3} c^{2}}\left[\left(\frac{4 G M}{r}-\boldsymbol{v}^{2}\right) \boldsymbol{r}+4(\boldsymbol{v} \cdot \boldsymbol{r}) \boldsymbol{v}\right]
$$

where $c$ is speed of light, and $\boldsymbol{r}, \boldsymbol{v}$ are heliocentric position and velocity vectors [5-7].

We used this code to benchmark problem i.e. the precession of Mercury's perihelion. This test gives good agreement with observation, and theory with a different of 1 arcseconds/century.

\section{Oblateness/quadrupole moment of the Sun $\left(J_{2}\right)$}

The Sun is not perfectly spherical, that is, oblateness of the Sun may influence the motion of the comet Lovejoy. We implement the quadrupole moment of the Sun in this simulation. We follow the derivation and program of this correction force of the Sun in mercury6 code [8]. Oblateness parameter $\left(J_{2}\right)=10^{-6}$ used in this simulation [9] and zero for $J_{4}$ and $J_{6}$. In newest REBOUND code, this correction force already provided in examples/ directory to study a dust orbiting Saturn. Oblateness correction force is very important in dynamics of satellite on Earth.

\section{Outgassing}

Marsden et al. (1973) [10] introduced what has become the standard in modeling cometary motion, i.e. symmetric nongravitational acceleration for comet due to outgassing. It is assumed that a cometary nucleus is undergo vaporization of water ice that acts symmetrically with respect to perihelion position [11]. These nongravitational accelerations expressed in (4) and (5).

$$
A_{1} g(r)(r / r)+A_{2} g(r) t+A_{3} g(r) \boldsymbol{n}
$$

$$
g(r)=\alpha\left(r / r_{o}\right)^{-m}\left(1+\left(r / r_{o}\right)^{n}\right)^{-k}
$$

The scale distance $r_{o}$ is the heliocentric distance where comet's ice surface start to sublimate. For water ice, $r_{o}=$ $2.808 \mathrm{AU}$ and the normalizing constant $\alpha=0.111262$. The exponents $m, n$, and $k$ equal to $2.15,5.093$, and 4.6142, respectively. The nongravitational acceleration is represented by a radial term, $A_{1} g(r)$, a transverse term, $A_{2} g(r)$, and normal term $A_{3} g(r)$ in the equations of motion. The radial unit vector $(r / r)$ is defined outward along the Sun-comet line, while the unit vector $(\boldsymbol{t})$ is directed normal to $(\boldsymbol{r} / r)$, in the orbit plane, and in the direction of the comet's motion. Normal term acceleration $A_{3} g(r)$ present for most active comets, but its periodic nature often makes it difficult to determine, and the value is very small. Because the constants of cometary outgassing components of this comet is not available, we adopted the ones belong to the known Sungrazing comet 96P/Machholz [12,13].

\section{RESULTS}
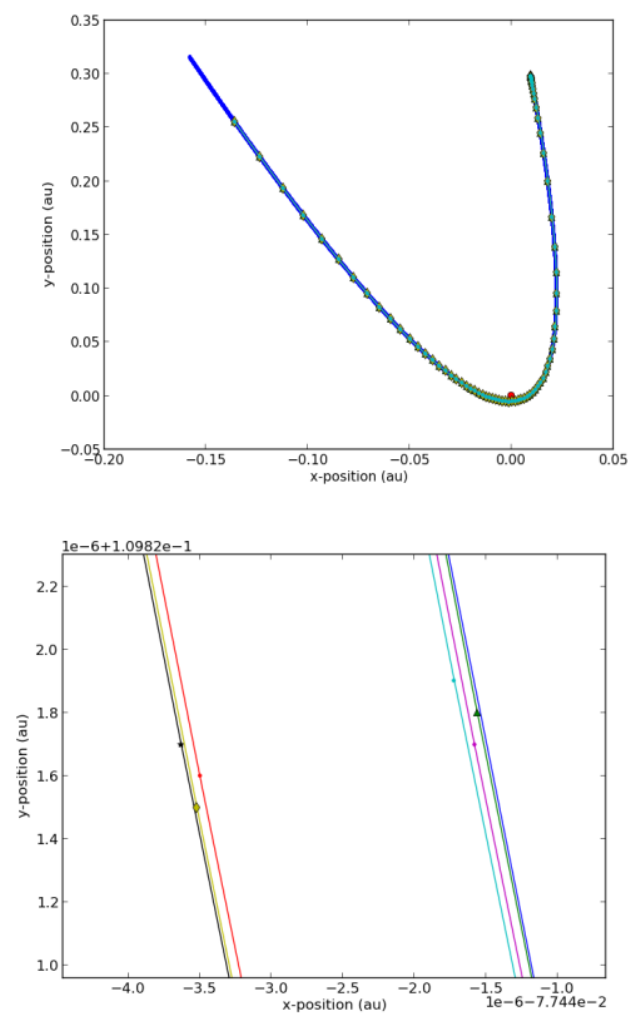

Figure 1. (upper panel) Position of comet Lovejoy in Cartesian coordinate ( $x y$-plane); red dot is Sun position, blue line is the orbit calculated by JPL NASA, and green dot is integration step using REBOUND, with and without additional forces. (lower panel). Position of "comet" in the red box calculated by different additional forces; red: only Newtonian gravity, yellow: oblateness, black: outgassing, green: relativistic correction, purple: relativistic correction + oblateness, cyan: relativistic correction + oblateness + outgassing, and blue: JPL NASA ephemeris.

where 
We present the result of motion of comet Lovejoy affected by additional forces in short dynamical time, i.e. encounter with the Sun and comeback time.

\section{A. Encounter with the Sun}

Orbit of Lovejoy a week before and after perihelion passage in Cartesian coordinate ( $x y$-plane) is presented in Fig. 1 (upper panel). Blue line is the orbit provided by JPL NASA, and appear as green dot is integration step, with and without additional forces.

We can identify how additional forces change the orbit by looking in detail of one step presented in Fig. 1 (lower panel). There are two group of data; left group is the orbit without implementation of relativistic correction and the right side group, which is closer to JPL data, is the orbit with implementation of relativistic correction to the equation of motion. When the comet Lovejoy reached perihelion the velocity is $565 \mathrm{~km} / \mathrm{s}(0.19 \%$ speed of light $)$ and the distance to the Sun is only $\sim 1.6$ radius of the Sun.

Impact of the oblateness correction force and symmetric outgassing parameter is very small to the orbit of comet during close encounter with the Sun. Model of outgassing process may very different if comet enter the corona of the Sun which happen in real condition of this comet Lovejoy. We present the change of semimajor axis of the comet during close encounter in Fig. 2. Orbit of the comet which is affected only by general relativity correction perfectly match with the data provided by JPL NASA.

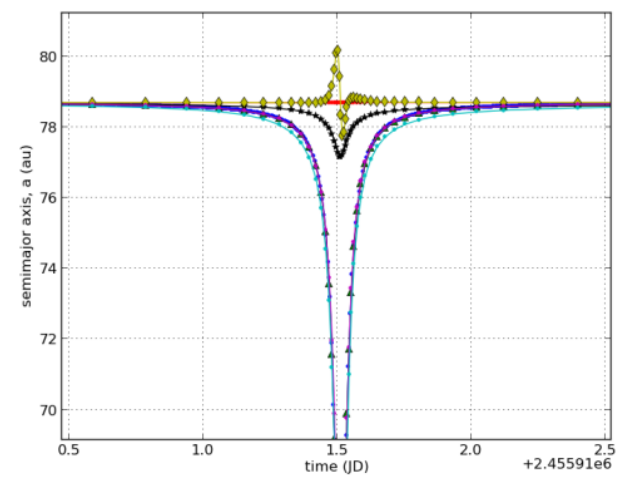

Figure 2. Semimajor axis during encounter with the Sun, color scheme is the same with Fig. 1.

\section{B. Comeback time}

Integration over 2000 years provide three comeback time after great passage in Dec. 2011 (period $\approx 622$ years). We present the change of semimajor axis of the comet for 2000 years in Fig. 3 (left). Effect of additional force on the first comeback time seen in Fig. 3 (right). Outgassing dominantly changes the comeback time of the comet $(\sim 150$ days using the adopted outgassing parameter). Outgassing in long dynamical time also changes the semimajor axis (longer $a$ seen in the first comeback time). This changes may affect the scenario of post-encounter, for example seen in the second encounter of this comet.
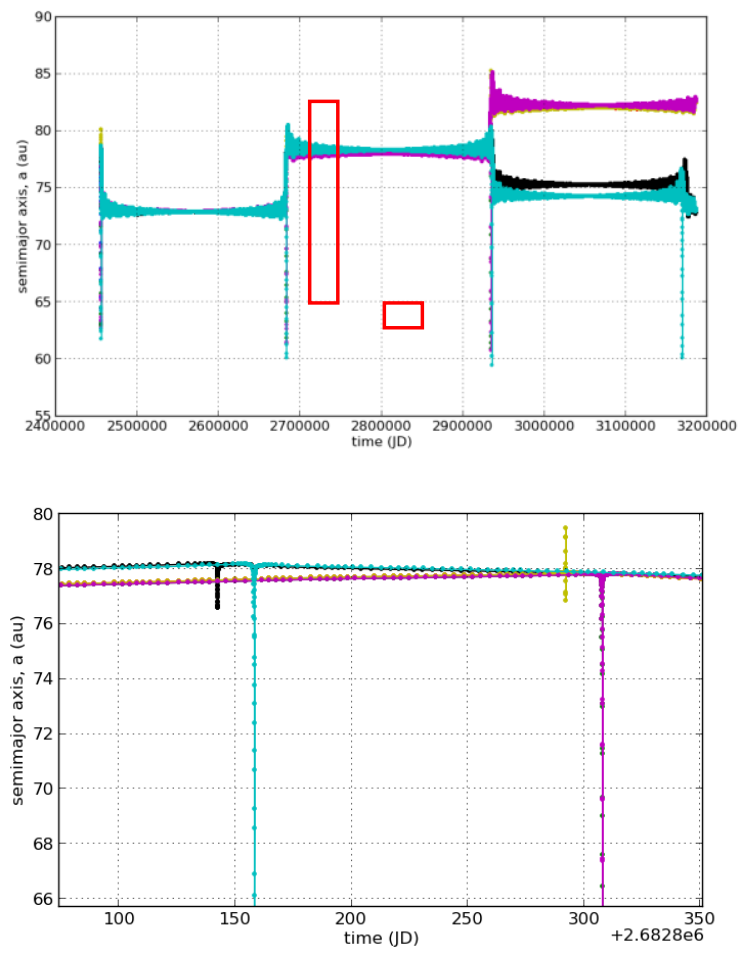

Figure 3. Left. Semimajor axis of comet Lovejoy for 2000 years. Right. First comeback of comet Lovejoy; outgassing parameter dominantly changes the comeback time (perihelion passage) presented as black and cyan which outgassing parameter is included in simulation. In long dynamical time outgassing also changes the semimajor axis; this may affect the post-encounter scenario as seen in second encounter of this comet.

\section{CONCLUSION}

We integrate the equations of motion of comet Lovejoy for 2000 years affected by outgassing, oblateness of the Sun, and relativistic correction. We implement this correction force in new REBOUND integrator. Relativistic correction plays important roles in encounter event of comet Lovejoy. Oblateness correction force has very small contribution to the equation of motion of comet. In long dynamical time, effect of symmetric outgassing parameter is more dominant; this effect may change the comeback time and semimajor axis of comet.

\section{ACKNOWLEDGMENT}

This work has been supported by ITB Research and Innovation Grant No. 1036m/I1.C01.2/PL/2014

\section{REFERENCES}

[1] Z. Sekanina, P. W. Chodas, "Comet C/2011 W3 (Lovejoy): Orbit Determination, Outbursts, Disintegration of Nucleus, Dust-Tail Morphology, and Relationship to New Cluster of Bright Sungrazers," Astrophys. J., vol. 757-127, May. 2012, pp. 1-33. 
[2] Z. Sekanina, P. W. Chodas, "Fragmentation Hierarchy of Bright Sungrazing Comets and the Birth and Orbital Evolution of the Kreutz System. II. The Case for Cascading Fragmentation,” Astron. J, vol. 663, Jul. 2007, pp. 657-676.

[3] H. Rein and S. F. Liu, "REBOUND: An Open-Source Multi-Purpose N-body Code for Collisional Dynamics," Astron. \& Astrophys., vol. 537-A128, pp. 1-10, 2012, doi:10.1051/0004-6361/201118085.

[4] H. Rein \& D. Spiegel, "Versatile High Order Integrator IAS15," 2014, unpublished.

[5] F. Benitez and T. Gallardo, "The Relativistic Factor in the Orbital Dynamics of Point Masses," Celest. Mech. Dyn. Astr., vol. 101, Jun. 2008, pp. 289-307, doi:10.1007/s10569-008-9146-5.

[6] J. D. Anderson, P. B. Esposito, W. Martin, C. L. Thornton, and D. O. Muhleman, "Experimental Test of General Relativity using TimeDelay Data from Mariner 6 and Mariner 7," Astrophys. J., vol. 200, Aug. 1975, pp. 221-233.

[7] T. Quinn, S. Tremaine, M. Duncan, "A Three Million Year Integration of the Earth's Orbit," Astron. J., vol. 101, 1991, pp. 22872305 .
[8] J. E. Chambers , "A Hybrid Symplectic Integrator that Permits Close Encounters between Massive Bodies," Mon. Not. R. Astron. Soc., vol. 304, Apr. 1999, pp. 793-799.

[9] L. Campbell, J. C. McDow, J. W. Moffat, and D. Vincent, "The Sun's Quadrupole Moment and Perihelion Precession of Mercury," Science, vol. 305, Oct. 1983, pp. 508-510.

[10] B. G. Marsden, Z. Sekanina, and D. K. Yeomans, "Comets and Nongravitational forces. V.,” Astron. J., vol. 78, 1973, pp. 211-225.

[11] D. K. Yeomans, P. W. Chodas, G. Sitarski, S. Szutowicz, and M Królikowska, Cometary orbit determination and nongravitational forces, In: Festou, M. C., Keller, H. U., Weaver, H. A. (eds.) Comets II, The University of Arizona Press, 2005, pp. 137-151.

[12] C. D. Murray and S. F. Dermott, Solar System Dynamics, New York: Cambridge Univ. Press, 2005, pp. 22-62.

[13] B. Dermawan et al., "Orbit of the Short-Lived Sun-Grazing Comet C/1999 X3," AIP Conference Proceedings 1589, pp. 14-17, 2014, doi:10.1063/1.4868739. 